\title{
Complex-scaling generalized pseudospectral method for quasienergy resonance states in two-center systems: Application to the Floquet study of charge resonance enhanced multiphoton ionization of molecular ions in intense low-frequency laser fields
}

\author{
Xi Chu and Shih-I Chu \\ Department of Chemistry, University of Kansas, and Kansas Center for Advanced Scientific Computing, Lawrence, Kansas 66045
}

(Received 22 April 2000; published 13 December 2000)

\begin{abstract}
We present a complex-scaling generalized pseudospectral method for accurate and efficient treatment of resonance states in two-center molecular systems, involving optimal nonuniform grid discretization of the Hamiltonian in prolate spheroidal coordinates. The procedure is applied to the first converged non-Hermitian Floquet study of multiphoton ionization of molecular ions in intense low-frequency $(1064 \mathrm{~nm})$ laser fields. We explore the underlying mechanism responsible for the ionization enhancement of $\mathrm{H}_{2}{ }^{+}$at some critical internuclear distances. Several features of the complex quasienergy states are observed. A detailed analysis of the nature and dynamical behavior of these quasienergy states reveals that the ionization enhancement is mainly due to the effect of charge-resonance-enhanced multiphoton resonances of the $1 \sigma_{g}$ and $1 \sigma_{u}$ states with excited electronic states at some particular internuclear distances. These "critical" distances depend on the details of molecular electronic structure and the laser frequency and intensity used in the study.
\end{abstract}

DOI: 10.1103/PhysRevA.63.013414

PACS number(s): 33.80.Rv, 33.80.Eh, 42.50.Hz, 33.90.+h

\section{INTRODUCTION}

The study of dissociative ionization of diatomic molecules in intense laser fields is a subject of considerable current interest both experimentally [1-5] and theoretically [6-8]. For the prototype molecular-ion system, $\mathrm{H}_{2}{ }^{+}$, both the time-dependent wavepacket method [6] and Floquet approach (using complex basis functions) $[7,8]$ have been used for the study of the multiphoton ionization in strong fields. Experimentally it has been found that linear molecules tend to align along the linear polarization of the laser fields. Further, the kinetic energy of the dissociated fragment ions appears to be independent of the laser pulse and ionization fraction, and is only a fraction of the Coulomb energy of the ions at the equilibrium separation $R_{e}[2,4,5]$. A possible interpretation of the latter observation is that "Coulomb explosion" does not take place at the equilibrium internuclear distance $R_{e}$ but at a larger "critical" distance $\left(R_{c}\right)$ at which the ionization rate peaks [9]. The enhanced ionization at some larger internuclear distance has been observed experimentally [3] and confirmed by theoretical consideration [6-8]. In the case of low-frequency ac fields, the theoretical analysis of the enhanced ionization process is generally proceeded by means of the over-the-barrier breakup mechanism in the static (dc) field limit at fixed internuclear distance $R$ [6-8]. However, there is still some disagreement on the the actual detailed mechanisms responsible for the enhanced ionization.

The goals of this paper are twofold: (a) First, we present the first converged non-Hermitian Floquet calculations of the multiphoton ionization of $\mathrm{H}_{2}{ }^{+}$(at fixed $R$ ) in the lowfrequency $(1064 \mathrm{~nm})$ regime and we explore the origin of ionization enhancement. Due to the large number of Floquet photon blocks involved in the low-frequency fields, "exact" Floquet calculations are difficult and previous studies of this problem have used either the cycle averaged rate (i.e., an average of the ionization rates at different dc fields over one optical cycle) $[7,8]$ or included the asymptotic expansion of the complex quasienergy to the $\omega^{2}$ order [8]. As will be shown later in this paper, the "exact" ac Floquet calculations reveal several new features on the characteristics and dynamical behavior of complex quasienergy states at different $R$, which have not been seen in the dc field limit. Examination of the nature of these complex quasienergy states leads to new insights on the detailed mechanisms for the enhanced ionization phenomenon. (b) Second, we introduce a new and general complex-scaling generalized pseudospectral (CSGPS) technique for the determination of resonance states, including complex quasienergy resonance states, for two-center diatomic molecular systems. In the present context, the CSGPS method provides an optimal nonuniform grid technique for the discretization of the molecular Floquet Hamiltonian. The CSGPS method was previously developed for the one-center atomic systems [10] and has been applied successfully to the study of multiphoton [11] and abovethreshold ionization [12] and high harmonic generation (HHG) [13] of neutral atoms or negative ions in strong fields. It was found that highly accurate results (for both ground and excited resonance states) can be efficiently obtained with the use of only a modest number of nonuniform pseudospectral grid points. The present extension of the CSGPS procedure to the two-center systems allows accurate and efficient determination of electronic quasienergy resonances of diatomic molecular systems in strong fields even for the challenging case of the low-frequency fields.

The paper is organized as follows. In Sec. II we outline the basic procedures for the determination of both bound and resonance states for two-center diatomic molecular systems with or without the external fields. In Sec. III, we extend the procedure along with the non-Hermitian Floquet formalism to the study of the phenomenon of the enhanced ionization of $\mathrm{H}_{2}{ }^{+}$in intense low-frequency $(1064 \mathrm{~nm})$ laser fields. The underlying mechanism of the ionization enhancement is then explored by means of a detailed study of the nature of the complex quasienergy states involved. 


\section{GENERALIZED PSEUDOSPECTRAL METHOD FOR THE BOUND AND RESONANCE STATES IN TWO-CENTER MOLECULAR SYSTEMS}

\section{A. Bound-state systems}

The field-free electronic Hamiltonian of $\mathrm{H}_{2}{ }^{+}$, in atomic units, can be written as

$$
\hat{H}=-\frac{1}{2} \nabla^{2}-\frac{1}{\left|\mathbf{r}-\mathbf{R}_{1}\right|}-\frac{1}{\left|\mathbf{r}-\mathbf{R}_{2}\right|},
$$

where $\mathbf{r}$ is the electronic coordinate, and $\mathbf{R}_{1}=(0,0, a)$ and $\mathbf{R}_{2}=(0,0,-\mathrm{a})$ are the coordinates of the two nuclei in Cartesian coordinates. The internuclear separation $R$ is equal to 2a. Now consider the bare electronic Hamiltonian in the prolate spheroidal coordinates $(\mu, \nu, \varphi), 0 \leqslant \mu<\infty$, $0 \leqslant \nu \leqslant \pi$, and $0 \leqslant \varphi \leqslant 2 \pi$, where $x=a \sinh \mu \sin \nu \cos \varphi$, $y=a \sinh \mu \sin \nu \sin \varphi$, and $z=a \cosh \mu \cos \nu$ [14]. Equation (1) can be recasted into the following form:

$$
\begin{aligned}
\hat{H}= & -\frac{1}{2 a^{2}}\left[\frac{1}{\left(\sinh ^{2} \mu+\sin ^{2} \nu\right) \sinh \mu} \frac{\partial}{\partial \mu}\left(\sinh \mu \frac{\partial}{\partial \mu}\right)\right. \\
& +\frac{1}{\left(\sinh ^{2} \mu+\sin ^{2} \nu\right) \sin \nu} \frac{\partial}{\partial \nu}\left(\sin \nu \frac{\partial}{\partial \nu}\right) \\
& \left.+\frac{1}{\sinh ^{2} \mu \sin ^{2} \nu} \frac{\partial^{2}}{\partial \varphi^{2}}\right]-\frac{2 \cosh \mu}{a\left(\cosh ^{2} \mu-\cos ^{2} \nu\right)} .
\end{aligned}
$$

Due to the axial symmetry of the system, the solutions of the static Schrödinger equation

$$
\hat{H} \psi=E \psi
$$

take the form,

$$
\psi_{m}(\mathbf{r})=e^{i m \varphi} \Phi(\mu, \nu), \quad(m=0, \pm 1, \pm 2 \ldots) .
$$

In the pseudospectral method, we expand $\Phi(\mu, \nu)$ by $\Phi_{N_{\mu}, N_{\nu}}(\mu, \nu)$, the polynomials of order $N_{\mu}$ and $N_{\nu}$ in $\mu$ and $\nu$, respectively,

$$
\begin{aligned}
\Phi(\mu, \nu) & =\Phi_{N_{\mu}, N_{\nu}}(\mu, \nu) \\
& =\sum_{i=0, j=0}^{N_{\mu}, N_{\nu}} \phi\left(\mu_{i}, \nu_{j}\right) g_{i}[x(\mu)] g_{j}[y(\nu)],
\end{aligned}
$$

and further require the approximation to be exact, i.e., $\Phi_{N_{\mu}, N_{\nu}}\left(\mu_{i}, \nu_{j}\right)=\phi\left(\mu_{i}, \nu_{j}\right) \equiv \phi_{i j}$, where $\left\{x\left(\mu_{i}\right)\right\} \quad$ and $\left\{y\left(\nu_{j}\right)\right\}$ are the two sets of collocation points to be described below. In Eq. (5), $g_{i}(x)$ and $g_{j}(y)$ are the cardinal functions $[10,11]$ defined as

$$
\begin{aligned}
& g_{i}(x)=-\frac{1}{N_{\mu}\left(N_{\mu}+1\right) P_{N_{\mu}}\left(x_{i}\right)} \frac{\left(1-x^{2}\right) P_{N_{\mu}}^{\prime}(x)}{x-x_{i}}, \\
& g_{j}(y)=-\frac{1}{N_{\nu}\left(N_{\nu}+1\right) P_{N_{\nu}}\left(y_{j}\right)} \frac{\left(1-y^{2}\right) P_{N_{\nu}}^{\prime}(y)}{y-y_{j}} .
\end{aligned}
$$

In the case of the Legendre pseudospectral method $[10,11]$, which we adopt in this article, the boundary points are $x_{0}$ $=y_{0}=-1 \quad$ and $x_{N_{\mu}}=y_{N_{\nu}}=1 . \quad x_{i}\left(i=1, \ldots, N_{\mu}-1\right) \quad$ and $y_{j}\left(j=1, \ldots, N_{\nu}-1\right)$ are the collocation points determined, respectively, by the roots of the first derivative of the Legendre polynomial $P_{N_{\mu}}$ with respect to $x$ and the first derivative of $P_{N_{\nu}}$ with respect to $y$, namely,

$$
\begin{aligned}
& P_{N_{\mu}}^{\prime}\left(x_{i}\right)=0, \\
& P_{N_{\nu}}^{\prime}\left(y_{j}\right)=0 .
\end{aligned}
$$

It follows that the cardinal functions possess the following unique properties:

$$
\begin{aligned}
& g_{i}\left(x_{i^{\prime}}\right)=\delta_{i, i^{\prime}}, \\
& g_{j}\left(y_{j^{\prime}}\right)=\delta_{j, j^{\prime}} .
\end{aligned}
$$

We shall use the following mapping relationships between $\mu$ and $\mathrm{x}$ and between $\nu$ and $\mathrm{y}$ :

$$
\begin{gathered}
\mu=L \frac{1+x}{1-x}, \\
\nu=\frac{\pi}{2}(1+y),
\end{gathered}
$$

where $x \in[-1,1], y \in[-1,1], \mu \in[0, \infty], \nu \in[0, \pi]$, and $L$ is the mapping parameter. The collocation points of $x(\mu)$ and $y(\nu)$ are determined by Eqs. (8) and (9). Having constructed the mesh structure, we define a set of discrete weights $w_{i}^{x}\left(i=0, \ldots, N_{\mu}\right), w_{j}^{y}\left(j=0, \ldots, N_{\nu}\right)$, and a pair of discrete matrices $d^{x}$ and $d^{y}$, which generate approximate integrals and partial derivatives on the mesh according to the relations (where $\mathrm{F}$ is a function of $x$ and $y$ ):

$$
\begin{aligned}
& \int_{-1}^{1} F d x \simeq \sum_{i} w_{i}^{x} F\left(x_{i}, y\right), \\
& \int_{-1}^{1} F d y \simeq \sum_{j} w_{j}^{y} F\left(x, y_{j}\right),
\end{aligned}
$$

and

$$
\begin{aligned}
& \left.\frac{\partial F}{\partial x}\right|_{x=x_{i}}=\sum_{k} d_{i k}^{x} F\left(x_{k}, y\right), \\
& \left.\frac{\partial F}{\partial y}\right|_{y=y_{j}}=\sum_{k} d_{j k}^{y} F\left(x, y_{k}\right),
\end{aligned}
$$

where

$$
d_{i k}^{x}=\left.\frac{d g_{k}(x)}{d x}\right|_{x=x_{i}},
$$




$$
d_{j k}^{y}=\left.\frac{d g_{k}(y)}{d y}\right|_{y=y_{j}} .
$$

Direct pseudospectral discretization of the Hamiltonian in Eq. (2) leads to an asymmetric eigenvalue problem. To symmetrize the Hamiltonian discretization, we consider the alternative but equivalent variational form of the Schrödinger equation

$$
\frac{\delta \int d^{3} \mathbf{r} \psi^{*}(\hat{H}-E) \psi}{\delta \psi^{*}}=0 .
$$

For the case of $\mathrm{H}_{2}{ }^{+}$in the prolate spheroidal coordinates, we rewrite the integral in Eq. (20) as [15]

$$
\begin{aligned}
F_{s} \equiv & \int d^{3} \mathbf{r} \psi^{*}(\hat{H}-E) \psi \\
= & \frac{1}{2 a^{2}} \int d^{3} \mathbf{r} \frac{1}{\sinh ^{2} \mu+\sin ^{2} \nu}\left[\left(\frac{\partial \Phi}{\partial \mu}\right)^{2}+\left(\frac{\partial \Phi}{\partial \nu}\right)^{2}\right] \\
& +\frac{m^{2}}{2 a^{2}} \int d^{3} \mathbf{r} \frac{\Phi^{2}}{\sinh ^{2} \mu \sin ^{2} \nu} \\
& -\int d^{3} \mathbf{r}\left[\frac{2 \cosh ^{2}}{a\left(\cosh ^{2} \mu-\cos ^{2} \nu\right)}+E\right]|\Phi|^{2}
\end{aligned}
$$

where

$$
d^{3} \mathbf{r}=a^{3}\left(\sinh ^{2} \mu+\sin ^{2} \nu\right) \sinh \mu \sin \nu d \mu d \nu d \varphi .
$$

Discretizing $F_{s}$ under the polynomial approximation, Eq. (5), we obtain

$$
\begin{aligned}
F_{s} \simeq & \sum_{i, i^{\prime}, j} \phi_{i^{\prime} j} \phi_{i j} \sum_{k} q_{k j} d_{k i}^{\mu} d_{k i^{\prime}}^{\mu}+\sum_{i, j, j^{\prime}} \phi_{i j^{\prime}} \phi_{i j} \sum_{l} q_{i l} d_{l j}^{\nu} d_{l j^{\prime}}^{\nu} \\
& +m^{2} \sum_{i, j} \phi_{i j}^{2} w_{i j}^{\prime}-\sum_{i, j} \phi_{i j}^{2} w_{i j}\left[\frac{2 \cosh \mu_{i}}{a\left(\cosh ^{2} \mu_{i}-\cos ^{2} \nu_{j}\right)}+E\right],
\end{aligned}
$$

where $\phi_{i j}$ stands for $\phi\left(\mu_{i}, \nu_{j}\right)$, and $q, w$, and $w^{\prime}$ are the weights

$$
\begin{gathered}
q_{i j}=2 \pi a w_{i}^{x} w_{j}^{y} \sinh \mu_{i} \sin \nu_{j}\left(\frac{d \mu}{d x}\right)_{x=x_{i}}\left(\frac{d \nu}{d y}\right)_{y=y_{j}}, \\
w_{i j}=q_{i j}\left(\sinh ^{2} \mu_{i}+\sin ^{2} \nu_{j}\right) \\
w_{i j}^{\prime}=w_{i j} / \sinh ^{2} \mu_{i} \sin ^{2} \nu_{j}
\end{gathered}
$$

and

$$
d_{i j}^{\mu}=\left(\frac{d x}{d \mu}\right)_{i} d_{i j}^{x}
$$

$$
d_{i j}^{\nu}=\left(\frac{d y}{d \nu}\right)_{i} d_{i j}^{y}
$$

It is now straightforward to perform the variation of $F_{s}$ with respect to $\psi$, Eq. (20), on the two-dimensional grid. This leads to a symmetric generalized eigenvalue problem:

$$
\begin{aligned}
& \left\{\frac{1}{2} \sum_{k, i^{\prime}} \phi_{i^{\prime} j} q_{k j} d_{k i}^{\mu} d_{k i^{\prime}}^{\mu}+\frac{1}{2} \sum_{j^{\prime}, l} \phi_{i j^{\prime}} q_{i l} d_{l j}^{\nu} d_{l j^{\prime}}^{\nu}\right\} \\
& +\left[w_{i j}^{\prime} m^{2}-\frac{2 w_{i j} \cosh \mu_{i}}{a\left(\cosh ^{2} \mu_{i}-\cos ^{2} \nu_{j}\right)}\right] \phi_{i j}=E w_{i j} \phi_{i j} .
\end{aligned}
$$

Equation (30) can be further simplified by setting $\chi_{i j}$ $=\sqrt{w}_{i j} \phi_{i j}[10]$ to obtain

$$
\begin{gathered}
\left\{\frac{1}{2} \sum_{k, i^{\prime}} \chi_{i^{\prime} j} \frac{q_{k j}}{\sqrt{w_{i^{\prime} j} w_{i, j}}} d_{k i}^{\mu} d_{k i^{\prime}}^{\mu}+\frac{1}{2} \sum_{j^{\prime}, l} \chi_{i j^{\prime}} \frac{q_{i l}}{\sqrt{w_{i j} w_{i j^{\prime}}}} d_{l j}^{\nu} d_{l j^{\prime}}^{\nu}\right\} \\
+\left[\frac{w_{i j}^{\prime}}{w_{i j}} m^{2}-\frac{2 \cosh \mu_{i}}{a\left(\cosh ^{2} \mu_{i}-\cos ^{2} \nu_{j}\right)}\right] \chi_{i j}=E \chi_{i j}
\end{gathered}
$$

This is the final working equation for the discretization of the $\mathrm{H}_{2}{ }^{+}$Hamiltonian in the prolate spheroidal coordinates. Equation (31) has the form of a standard eigenvalue problem of a sparse real symmetric matrix.

As a measure of the accuracy of the procedure, we have calculated the ground-state energy of $\mathrm{H}_{2}{ }^{+}$. Using only a modest number of grid points (12 points in $\mu$ and 10 points in $\nu$ ), we have obtained $E=-1.1026342144949$ a.u., in complete agreement with the exact value -1.1026342144949 a.u [16].

\section{B. Complex-scaling generalized pseudospectral method for the resonance-state problems}

The generalized pseudospectral method described above for bound-state eigenvalue problems can be extended to the resonance-state complex eigenvalue problems by means of the complex-scaling transformation [17]. In the prolate spheroidal coordinates, only the coordinate $\mu$ needs to be complex rotated, namely,

$$
\mu \rightarrow \mu e^{i \alpha},
$$

where $\alpha$ is the rotation angle. Consider, for example, the dc field ionization of $\mathrm{H}_{2}{ }^{+}$. In a static electric field parallel to the molecular $(\hat{\mathbf{z}})$ axis, the Hamiltonian of $\mathrm{H}_{2}{ }^{+}$becomes

$$
\hat{H}=-\frac{1}{2} \nabla^{2}-\frac{2 \cosh \mu}{a\left(\cosh ^{2} \mu-\cos ^{2} \nu\right)}+F a \cosh \mu \cos \nu,
$$

where $F$ is the electric-field amplitude. Following the similar discretization procedures as discussed before, we obtain the following sparse complex symmetric matrix eigenvalue problem: 
TABLE I. The calculated de width $\Gamma_{1 \sigma_{g}}$ and $\Gamma_{1 \sigma_{u}}$ of the $1 \sigma_{g}$ and $1 \sigma_{u}$ energy levels of $\mathrm{H}_{2}^{+}$. Also shown for comparison are the results obtained by Zuo and Bandrauk [6] and Mulyukov et al., [8]. The dc-field strength used is $F=0.0533$ a.u.

\begin{tabular}{|c|c|c|c|c|c|c|}
\hline & & $\Gamma_{1 \sigma_{g}}$ (a.u.) & & & $\Gamma_{1 \sigma_{u}}$ (a.u.) & \\
\hline$R$ (a.u.) & Ref. [6] & Ref. [8] & Present & Ref. [6] & Ref. [8] & Present \\
\hline 6 & $2.2 \times 10^{-6}$ & $5.69 \times 10^{-6}$ & $5.692 \times 10^{-6}$ & $9.8 \times 10^{-4}$ & $1.87 \times 10^{-3}$ & $1.873 \times 10^{-3}$ \\
\hline 10 & $1.3 \times 10^{-5}$ & $3.92 \times 10^{-5}$ & $3.922 \times 10^{-5}$ & $1.5 \times 10^{-3}$ & $2.20 \times 10^{-3}$ & $2.197 \times 10^{-3}$ \\
\hline 14 & $2.5 \times 10^{-5}$ & $7.30 \times 10^{-6}$ & $7.305 \times 10^{-6}$ & $2.8 \times 10^{-4}$ & $6.78 \times 10^{-4}$ & $6.778 \times 10^{-4}$ \\
\hline
\end{tabular}

$$
\begin{aligned}
& \frac{1}{2} \sum_{k, i^{\prime}} \chi_{i^{\prime} j} \frac{q_{k j}}{\sqrt{w_{i^{\prime} j} w_{i, j}}} d_{k i}^{\mu} d_{k i^{\prime}}^{\mu}+\frac{1}{2} \sum_{j^{\prime}, l} \chi_{i j^{\prime}} \frac{q_{i l}}{\sqrt{w_{i j} w_{i j^{\prime}}}} d_{l j}^{\nu} d_{l j^{\prime}}^{\nu} \\
& \quad+\left(F a \cosh \mu_{i} \cos \nu_{j}-\frac{2 \cosh \mu_{i}}{a\left(\cosh ^{2} \mu_{i}-\cos ^{2} \nu_{j}\right)}\right) \chi_{i j}=E \chi_{i j} .
\end{aligned}
$$

Some results of our calculated dc field ionization widths are presented in Table I. Also shown in Table I are the results obtained by Mulyukov et al., [8] using a different timeindependent method. The agreement of these two timeindependent calculations is excellent. The $\mathrm{dc}$ ionization widths of $\mathrm{H}_{2}{ }^{+}$have also been calculated by Zuo and Bandrauk [6] by a time-dependent procedure. Their estimates (shown in Table I) are smaller than the two time-independent calculations by a factor of 2 to 3 .

\section{Complex-scaling generalized pseudospectral method}

for the determination of complex quasienergies associated with multiphoton ionization processes

Corresponding to the periodically time-dependent Hamiltonian, in atomic units,

$$
\begin{aligned}
\hat{H}(\mathbf{r}, t)= & -\frac{1}{2} \nabla^{2}-\frac{2 \cosh \mu}{a\left(\cosh ^{2} \mu-\cos ^{2} \nu\right)} \\
& +a F \cosh \mu \cos \nu \cos \omega t,
\end{aligned}
$$

describing the interaction of the $\mathrm{H}_{2}{ }^{+}$molecule with a monochromatic, linearly polarized, coherent laser field of frequency $\omega$ and peak field strength $F$, with electric field along the internuclear axis $\hat{z}$, one can look for the wave function $\Psi(t)$ in the following form according to Floquet theorem [18]:

$$
\Psi(\mathbf{r}, t)=e^{-i \epsilon t} \Phi(\mathbf{r}, t) .
$$

Here $\epsilon$ is the quasienergy, and $\Phi(\mathbf{r}, t)$ is time periodic and can be expanded in a Fourier series with the fundamental frequency $\omega$ :

$$
\Phi(\mathbf{r}, t)=\sum_{n} \Phi_{n}(\mathbf{r}) e^{-i n \omega t}
$$

where the quasienergy-state Fourier component $\Phi_{n}(\mathbf{r})$ is time independent. An equivalent time independent Floquet Hamiltonian $\hat{H}_{F}$ may be obtained by [18]

$$
\begin{gathered}
{\left[\hat{H}_{0}-\epsilon-n \omega\right] \Phi_{n}+\frac{1}{2} F a \cosh \mu \cos \nu\left[\Phi_{n-1}+\Phi_{n+1}\right]=0} \\
(n=0, \pm 1, \pm 2, \ldots) .
\end{gathered}
$$

Performing the complex-scaling transformation, Eq. (32), and using the variational formulation [similar to Eq. (20)] for the Floquet Hamiltonian, it leads to the minimization of

$$
\begin{gathered}
F_{s}=\frac{2}{R^{2}} \int d^{3} \mathbf{r} \frac{1}{\sinh ^{2} \mu+\sin ^{2} \nu}\left[\left(\frac{\partial \Phi_{n}}{\partial \mu}\right)^{2}+\left(\frac{\partial \Phi_{n}}{\partial \nu}\right)^{2}\right] \\
-\int d^{3} \mathbf{r}\left[\frac{2 \cosh \mu}{a\left(\cosh ^{2} \mu-\cos ^{2} \nu\right)}+n \omega+\epsilon\right]\left(\Phi_{n}\right)^{2} \\
+F a \int d^{3} \mathbf{r} \cosh \mu \cos \nu\left(\Phi_{n-1}+\Phi_{n+1}\right) \Phi_{n}, \\
(n=0, \pm 1, \pm 2, \ldots) .
\end{gathered}
$$

Discretizing Eqs. (39) by means of the CSGPS method and performing the minimization, we obtain

$$
\begin{gathered}
\frac{1}{2} \sum_{k, i^{\prime}} \chi_{i^{\prime} j}^{n} \frac{q_{k j}}{\sqrt{w_{i^{\prime} j} w_{i, j}}} d_{k i}^{\mu} d_{k i^{\prime}}^{\mu}+\frac{1}{2} \sum_{j^{\prime}, l} \chi_{i j^{\prime}}^{n} \frac{q_{i l}}{\sqrt{w_{i j} w_{i j^{\prime}}}} d_{l j}^{\nu} d_{l j^{\prime}}^{\nu} \\
+F a \cosh \mu_{i} \cos \nu_{j}\left(\chi_{i j}^{n-1}+\chi_{i j}^{n+1}\right) \\
-\frac{2 \cosh \mu_{i}}{a\left(\cosh ^{2} \mu_{i}-\cos ^{2} \nu_{j}\right)} \chi_{i j}^{n}=\epsilon \chi_{i j}^{n}, \\
(n=0, \pm 1, \pm 2, \ldots) .
\end{gathered}
$$

Then we have an infinite dimensional complex symmetric matrix, whose complex eigenvalues $\left[\epsilon=\left(E_{R},-\Gamma / 2\right)\right]$ are related to the positions and widths of the shifted and broadened complex quasienergy states [18].

\section{A CASE STUDY: NON-HERMITIAN FLOQUET STUDY OF THE CHARGE-RESONANCE-ENHANCED MULTIPHOTON IONIZATION OF $\mathrm{H}_{2}^{+}$IN THE INTENSE LOW-FREQUENCY LASER FIELDS}

In this section, we present an application of the CSGPS procedure along with the non-Hermitian Floquet formalism [18] to the study of the mechanisms for the enhanced ionization phenomenon of $\mathrm{H}_{2}^{+}$ions in intense monochromatic low-frequency laser fields. The laser parameters used are 


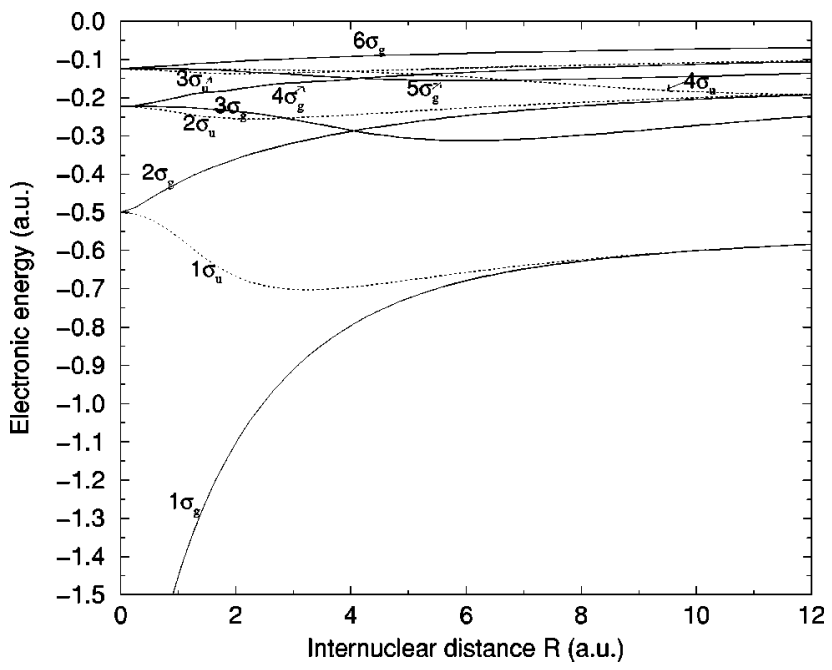

FIG. 1. Electronic energy levels of $\Sigma$ electronic states of fieldfree $\mathrm{H}_{2}^{+}$molecular ion vs internuclear distance $R$. The protonproton Coulomb repulsion energy is not included. The n $\sigma_{g}$ states are in solid lines, while the $\mathrm{n} \sigma_{u}$ states are in dotted lines.

wavelength $1064 \mathrm{~nm}$ and peak intensity $10^{14} \mathrm{~W} / \mathrm{cm}^{2}$. Assuming the laser polarization is along the internuclear $\left(\hat{z}^{-}\right)$ axis, only the $\Sigma$ electronic states need to be considered.

Figure 1 shows the field-free electron energy levels of $\mathrm{H}_{2}{ }^{+}$as a function of internuclear distance $R$, obtained by the generalized pseudospectral method discussed in Sec. II. The two lowest electronic states, $1 \sigma_{g}$ and $1 \sigma_{u}$, become nearly degenerate at larger $R$. In the presence of the external fields, the electric dipole coupling of $1 \sigma_{g}$ and $1 \sigma_{u}$ is linearly proportional to $R$ and becomes very significant. This phenomenon, known as the "charge resonance" (CR) effect [19], occurs only in the odd-charged molecular-ion systems. As will be exploited in this section, the combined effect of CR and the multiphoton transitions to excited electronic states is the main mechanism responsible for the enhanced ionization phenomenon observed for the molecular-ion systems.

In Figs. 2 and 3, we present the $R$-dependent real and imaginary parts of the complex quasienergies of $\mathrm{H}_{2}^{+}$, respectively, in the presence of the linearly polarized 1064-nm monochromatic laser field with peak intensity $10^{14} \mathrm{~W} / \mathrm{cm}^{2}$. The number of grid points used are 46 in the $\mu$ coordinate and 34 in the $\nu$ coordinate. Due to the symmetry of the system, only half of the $\nu$ grid points are actually needed. Up to 121 Floquet photon blocks are used to achieve fully converged results. The largest dimension of the Floquet matrix considered in this study is 87120 . The complex quasienergy eigenvalues can be determined accurately and efficiently by the implicitly restarted Arnoldi algorithm for a complex sparse matrix previously developed and used for the atomic resonances [11]. To our knowledge, this is the first successful attempt to perform fully converged Floquet calculations for low-frequency $(1064 \mathrm{~nm})$ multiphoton processes in molecular systems.

Due to the large number of the electronic and Floquet blocks involved, the resulting Floquet energy-level structure is rather complicated. In Fig. 2, we show only those quasienergy states whose major components are the field-free $1 \sigma_{g}$

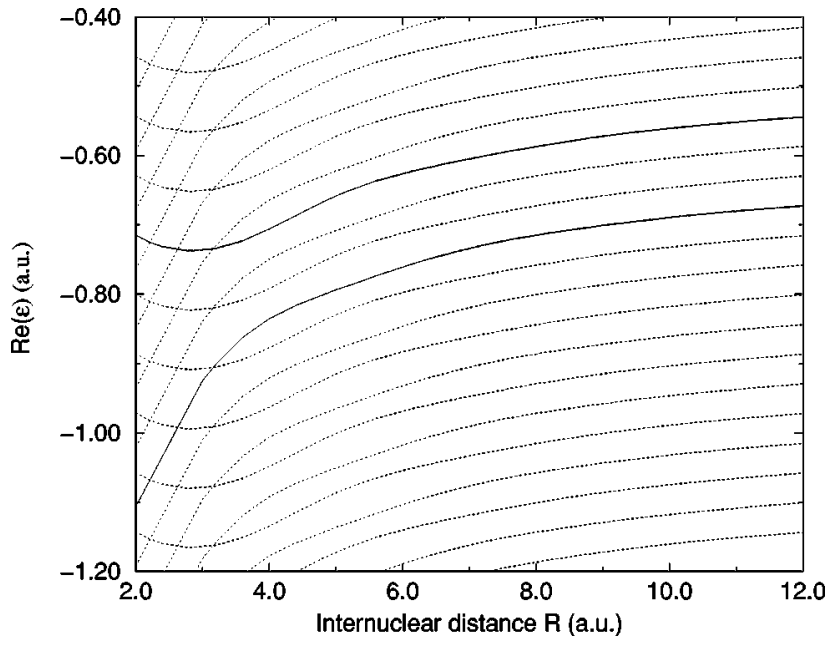

FIG. 2. The real parts of the complex quasienergies vs $R$. Only those quasienergy states whose dominant components are from $1 \sigma_{g}$ and $1 \sigma_{u}$ states are included. Two different groups of quasienergy states can be identified. The solid lines indicate one representative quasienergy level from each group.

and $1 \sigma_{u}$ states. In fact, the information regarding enhanced ionization can be extracted from these complex quasienergy states alone. Fig. 2 shows several intriguing behaviors of the real parts of the quasienergy levels. Firstly, the quasienergy levels are nearly parallel to each other in the larger internuclear separation regime, $R>6 \mathrm{a}_{0}$, and the separation of each quasienergy curve is equal to the photon frequency $\omega$. On the other hand, the behavior is quite different at shorter distance $\left(R<5 a_{0}\right)$. For $R$ close to the equilibrium distance $\left(R_{e}\right)$, the quasienergy levels are separated into two different groups. In one group, $\operatorname{Re}(\epsilon)$ first increases with increasing $R$ and resembles the behavior of the field-free $1 \sigma_{g}$ state. (We shall call this group the "lower' group.) In the other group, $\operatorname{Re}(\epsilon)$ first decreases with increasing $R$ and resembles the behavior of the field-free $1 \sigma_{u}$ state. (We shall call this group the "upper' group.) Within each group, the separation of

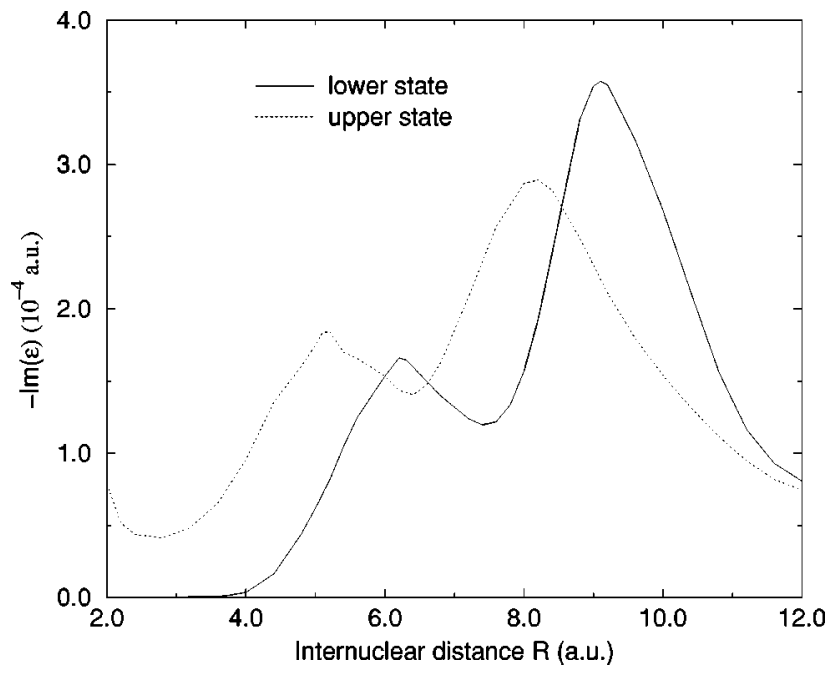

FIG. 3. The imaginary parts of the complex quasienergies vs $R$ from the lower and upper groups of quasienergy levels. 
nearby quasienergy levels are exactly $2 \omega$. We label two of the representative quasienergy levels (one from each group) by solid lines in Fig. 2. An important consequence of the Floquet symmetry is that all of these quasienergy states in the lower (upper) group, separated by $2 m \omega$ ( $m$ integer) in energy, are in fact physically indistinguishable and contain the same information regarding multiphoton dynamics. Thus, for example, while the (real) energy is separated by $2 m \omega$, each quasienergy state in the lower (or upper) group has the identical imaginary energy, $\operatorname{Im}(\epsilon)$, since the latter is related to the total ionization rate of the physical state. Thus in the following we shall use the dynamical information contained in the two quasienergy levels (denoted by solid lines in Fig. 2) to explore the multiphoton dynamics and the mechanisms responsible for the enhanced ionization phenomenon.

Figure 3 shows the $R$-dependent imaginary parts (widths) of the complex quasienergies in the lower (solid line) and upper (dotted line) groups, respectively. It is intriguing that both curves exhibit two major peaks in the ionization rate at certain larger distances $R$. For the "lower" group, the largest ionization enhancement occurs at $R \simeq 9 \mathrm{a}_{0}$ and a second enhancement occurs around $R \simeq 6.2 \mathrm{a}_{0}$. For the upper group, the major enhancement occurs around $R \simeq 8 \mathrm{a}_{0}$ and the second enhancement at $R \simeq 5 \mathrm{a}_{0}$. The ionization enhancement phenomenon at some larger internuclear distance $R$ has been reported in other recent theoretical studies [6-8] and interpreted in the light of the over-the-barrier ionization picture in the dc-field limit. In the dc field, the ionization width of the upper " $2 p$ " level (in the field-free united-atom language) is much larger than that of the lower " $1 s$ " [8] state. In a lowfrequency laser field, a certain amount of the electron population is excited to the $2 p$ level due to charge resonance and/or multiphoton absorption. Thus in the dc-field picture, the $2 p$ level is considered to be the major state responsible for the observed ionization enhancement [6-8]. Zuo and Bandrauk [6] argued that the major ionization peak arises from over-the-barrier ionization of the $2 p$ level out of the higher electronic-field potential well. Mulyukov et al., [8] on the other hand, have suggested that the ionization enhancement is due to the mixing of the $2 p$ state, which is localized in the higher well of the double-well electronic-field potential, with energetically nearby highly excited states that are localized in the lower potential well. Over-the-barrier ionization from the lower well can proceed without the impediment of back scattering of the electron from the hump between the wells. These authors also performed perturbative corrections, through order $\omega^{2}$, of the shifts of $1 s$ and $2 p$ levels in the low-frequency ac field [8]. However, the perturbative corrections breakdown near ionization peak positions. Although the dc-field picture of the ionization enhancement is insightful, detailed mechanism does not yet appear to be settled. Also the dc-field predicted peak positions are somewhat different from those of our quasienergy calculations. Finally, our results indicate that the quasienergy states in both upper and lower groups both show double-peak enhancement features. Qualitatively, this latter observation may be attributed to the fact that our Floquet study is a genuine ac-field study and the upper and lower quasienergy states are the dynamical combination of both the " $1 s$ " and " $2 p$ " levels in the sense

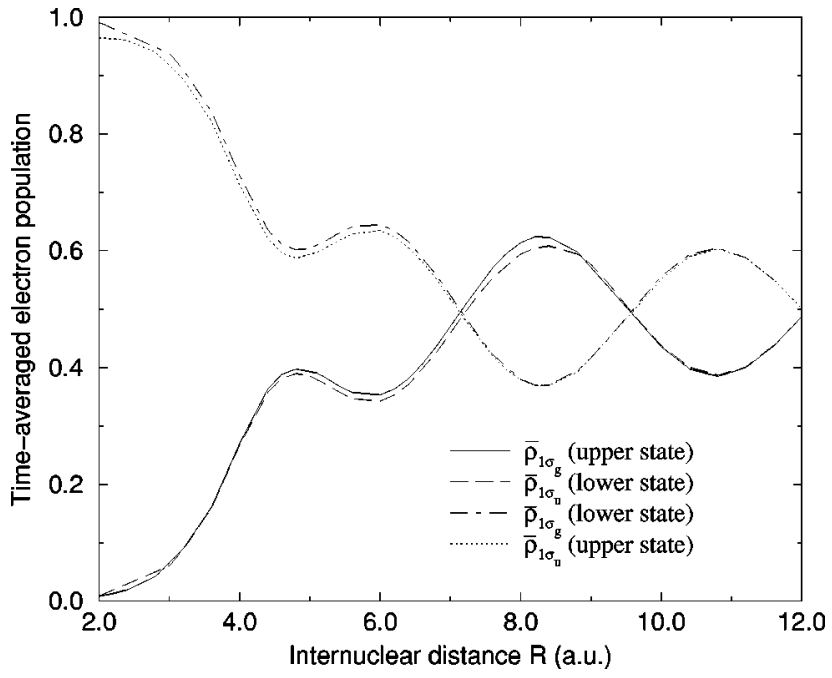

FIG. 4. The time-averaged electron population in $1 \sigma_{g}$ and $1 \sigma_{u}$ states from the lower and upper groups of quasienergy states.

that the majority of the electron population is transferring back and forth between the field-free $1 \sigma_{g}$ and $1 \sigma_{u}$ states. A more detailed analysis will be presented below.

It is instructive to study the property and dynamical behavior of these two groups of Floquet quasienergy states in the low-frequency and charge resonance regimes as a function of internuclear distance $R$. We consider first the timeaveraged electron population remaining in the bare $1 \sigma_{g}$ and $1 \sigma_{u}$ states:

$$
\begin{aligned}
& \bar{\rho}_{1 \sigma_{g}}^{k}=\frac{1}{T} \int_{0}^{T} d t\left|\sum_{n} e^{-i n \omega t}\left\langle 1 \sigma_{g} \mid \Phi_{n}^{k}\right\rangle\right|^{2}=\sum_{n}\left|\left\langle 1 \sigma_{g} \mid \Phi_{n}^{k}\right\rangle\right|^{2}, \\
& \bar{\rho}_{1 \sigma_{u}}^{k}=\frac{1}{T} \int_{0}^{T} d t\left|\sum_{n} e^{-i n \omega t}\left\langle 1 \sigma_{u} \mid \Phi_{n}^{k}\right\rangle\right|^{2}=\sum_{n}\left|\left\langle 1 \sigma_{u} \mid \Phi_{n}^{k}\right\rangle\right|^{2},
\end{aligned}
$$

where T $=2 \pi / \omega$ is the period, $\Phi_{n}$ is the nth Fourier component of the quasienergy state from Eq. (37), and $k$ is the index for different Floquet states corresponding to those adiabatic quasienergy levels shown in Figure 2. Note that here we only consider the periodic part of the quasienergy eigenfunctions, Eq. (37), since the quasienergy exponential factor $e^{-\Gamma t}$, Eq. (36), describes only the overall decaying behavior. The results are shown in Fig. 4 for both the upper and lower quasienergy states (namely, those denoted by solid lines in Fig. 2). In fact all the upper-group quasienergy states, separated by $2 m \omega$ ( $m$ integer), have the identical electron population behavior as that shown in Fig. 4 and is independent of the index $k$. It is the same for all the quasienergy states in the lower group. We found that $\bar{\rho}_{1 \sigma_{g}}^{k}+\bar{\rho}_{1 \sigma_{u}}^{k}$ $\simeq 1$, indicating that the dominant electron population still remains in the $1 \sigma_{g}$ and $1 \sigma_{u}$ states in this laser intensity $\left(10^{14}\right.$ $\left.\mathrm{W} / \mathrm{cm}^{2}\right)$. Moreover, the curve for $\bar{\rho}_{1 \sigma_{g}}$ in the upper state is almost the same as that of $\bar{\rho}_{1 \sigma_{u}}$ in the lower state, and vice versa. Thus the upper and lower quasienergy states form a 


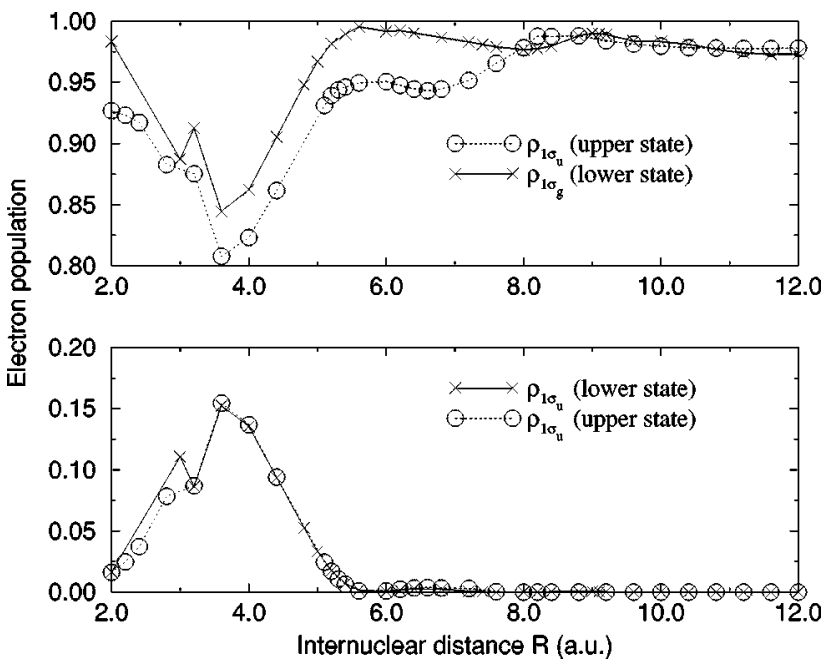

FIG. 5. The $R$-dependent electron population in the $1 \sigma_{g}$ and $1 \sigma_{u}$ states at time $t=m \pi / \omega$, where $m$ is a positive integer. The population is independent of $m$.

pair of complementary states. Finally it is interesting to see the oscillatory behavior of the electron population as a function of $R$ at larger internuclear separation.

We also examine the temporal behavior of electronic population at different time as a function of internuclear separation $R$. Again we only consider the periodic part of the quasienergy eigenfunctions of typical upper and lower states. In Fig. 5 we show the electron population remaining in the $1 \sigma_{g}$ and $1 \sigma_{u}$ states at $t=m \pi / \omega$, corresponding to the peak field amplitude case, where $m$ is a positive integer:

$$
\begin{aligned}
& \rho_{1 \sigma_{g}}\left(t=\frac{m \pi}{\omega}\right)=\left|\sum_{n} e^{-i n m \pi}\left\langle 1 \sigma_{g} \mid \Phi_{n}\right\rangle\right|^{2}, \\
& \rho_{1 \sigma_{u}}\left(t=\frac{m \pi}{\omega}\right)=\left|\sum_{n} e^{-i n m \pi}\left\langle 1 \sigma_{u} \mid \Phi_{n}\right\rangle\right|^{2} .
\end{aligned}
$$

We found that at this instant of time (in each optical cycle, regardless the value of $m$ ), the dominant population is in the $1 \sigma_{g}$ level for the lower quasienergy state and in the $1 \sigma_{u}$ level for the upper quasienergy state. This is partly due to the fact that at $t=0$, the lower state reduces to the fieldfree $1 \sigma_{g}$ level while the upper state reduces to the field-free $1 \sigma_{u}$ level. Several salient features are observed in Fig. 5. First, we see a significant "dip" in the electron population nearby $R \simeq 3.6 \mathrm{a}_{0}$. Notice that the energy separation (at fixed $R$ ) of the bare $1 \sigma_{g}$ and $1 \sigma_{u}$ levels (Fig. 1) is larger than one-photon energy for $R<5.1 a_{0}$. Thus in these shorter internuclear distance ranges, the transition between these two states is multiphoton dominant. The dip around $R \simeq 3.6 \mathrm{a}_{0}$ indicates that there is a multiphoton resonance between the $1 \sigma_{g}$ and $1 \sigma_{u}$ levels and some population transfer between the two levels takes place. When the internuclear separation $R$ becomes larger than $6 \mathrm{a}_{0}$, however, the $1 \sigma_{g}$ and $1 \sigma_{u}$ levels becomes nearly degenerate and the electric dipole coupling between the two levels is significant and increases linearly with increasing $R$. The quasienergy eigenfunction now

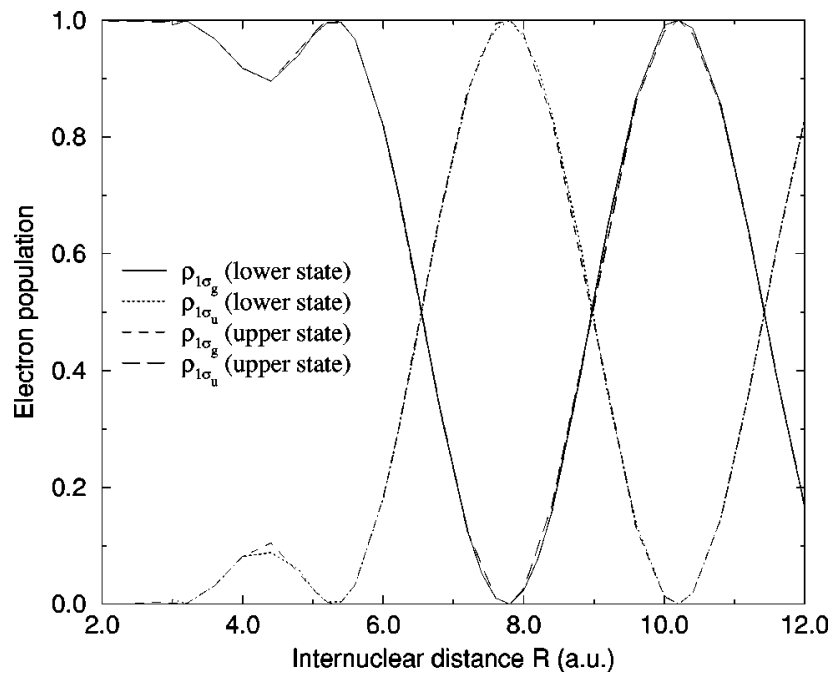

FIG. 6. Same as that of Fig. 5 except that the time is now set at $t=(2 m+1) \pi / \omega$.

spreads among many Fourier components. In this case, the modulus of the exponential time factor $e^{-i n m \pi}$ is unity, and all the $1 \sigma_{g}$ (or $1 \sigma_{u}$ ) Fourier components have the same sign and they add up constructively. As a result, there is little "net" electron transfer between the $1 \sigma_{g}$ and $1 \sigma_{u}$ levels and the electron population remains close to unity in the $1 \sigma_{g}$ level for the lower quasienergy state and in the $1 \sigma_{u}$ level for the upper quasienergy state for larger $R$.

The electron population behavior at $t=(2 m+1) \pi / 2 \omega, m$ integer, corresponding to the instant (in each optical) of zero electric dipole coupling, is shown in Fig. 6:

$$
\begin{aligned}
& \rho_{1 \sigma_{g}}\left(t=\frac{(2 m+1) \pi}{2 \omega}\right)=\left|\sum_{n} e^{-i n(2 m+1) \pi / 2}\left\langle 1 \sigma_{g} \mid \Phi_{n}\right\rangle\right|^{2}, \\
& \rho_{1 \sigma_{u}}\left(t=\frac{(2 m+1) \pi}{2 \omega}\right)=\left|\sum_{n} e^{-i n(2 m+1) \pi / 2}\left\langle 1 \sigma_{u} \mid \Phi_{n}\right\rangle\right|^{2} .
\end{aligned}
$$

In this case, the exponential time factor $e^{-i n(2 m+1) \pi / 2}$ becomes $+1(+\mathrm{i})$ and $-1(-\mathrm{i})$ alternatively for the $1 \sigma_{g}\left(1 \sigma_{u}\right)$ Fourier components, resulting in the near cancellation of the contributions from various Fourier components. The outcome is that electron absorbs and emits photons and jump back and forth between the $1 \sigma_{g}$ and $1 \sigma_{u}$ Floquet ladders as $R$ varies. For larger $R$, the electron populations show a near periodic function of $R$ and the upper and lower states form a pair of conjugated levels.

When all the time within a given optical cycle is considered, the averaged electron population behavior is depicted in Fig. 4. The oscillatory behavior at larger $R$ regime can be described as the consequence of a "charge-resonanceenhanced multiphoton transition" effect, namely, strong $1 \sigma_{g}-1 \sigma_{u}$ coupling prevails in each sequential photon absorption/emission in the Floquet state ladders: 


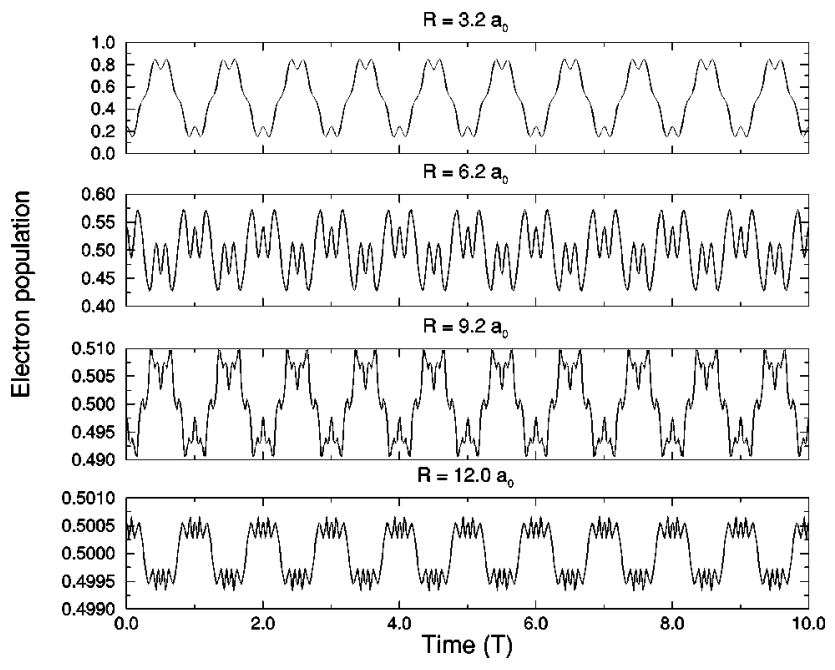

FIG. 7. The temporal behavior of the electron population of the lower quasienergy states at several internuclear separations $R$. Only the population in the "right"'-half space $(z>0)$ is included.

$$
\begin{gathered}
\ldots\left|1 \sigma_{g}, n>\Leftrightarrow\right| 1 \sigma_{u}, n-1>\Leftrightarrow\left|1 \sigma_{g}, n-2>\Leftrightarrow\right| 1 \sigma_{u}, \\
n-3>\Leftrightarrow \mid 1 \sigma_{g}, n-4>\ldots,
\end{gathered}
$$

where $n$ is the photon block index and $\Leftrightarrow$ denotes the electric dipole coupling. Since the $1 \sigma_{g}$ and $1 \sigma_{u}$ levels are nearly degenerate, the coupling in each step is strong. The resulting quasienergy eigenfunction spreads among many higher Fourier components.

It is also instructive to examine the time evolution of the electron population (at fixed internuclear distance $R$ ) at each electronic-field potential well, providing the information about the electron localization and/or delocalization. Since we are dealing with the ac field problem, the population in the left $(z<0)$ and right $(z>0)$ potential wells should be identical. This population can be estimated by integrating the electron population over half of the space (say, the right-half space, $z>0$ ) only

$$
\begin{aligned}
n_{\text {right }}(t)= & 2 \pi a^{3} \int_{0}^{\frac{\pi}{2}} d \nu \int_{0}^{\infty} d \mu\left(\sinh ^{2} \mu+\sin ^{2} \nu\right) \\
& \times \sinh \mu \sin \nu \Phi(\mu, \nu, t),
\end{aligned}
$$

where $\Phi(\mu, \nu, t) \equiv \Phi(\mathbf{r}, t)$ in Eq. (36). Again we consider here only the periodic part of the quasienergy eigenfunction. Several representative internuclear distances $R$ are chosen to display the electron population behavior in the right potential well $(z>0)$ : those two distances that exhibit the ionization peak positions and one smaller and one larger $R$. The results are shown for the lower (upper) quasienergy state in Fig. 7 (Fig. 8). Note that $\mathrm{n}_{\text {right }}(t)$ is a periodic function of time as it should be. The most prominent feature is that it fluctuates up and down around the mean value 0.5 for all the distance $R$. Thus the electron does not appear to prefer to localize in the right $(z>0)$ or left $(z<0)$ potential well. This ac picture is different from that inferred from the dc picture. Notice also that the amplitude of the fluctuation decreases as $R$ increases.

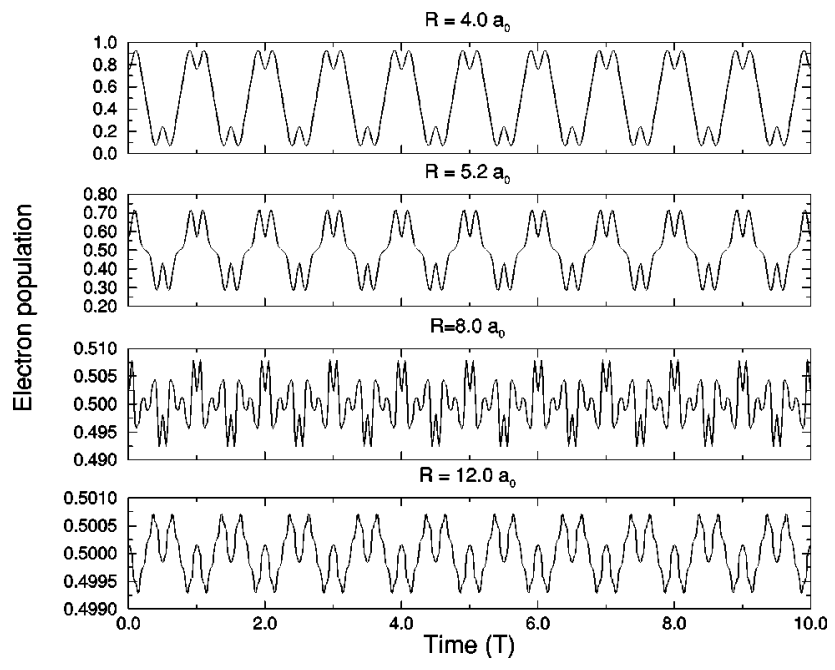

FIG. 8. Same as those of Fig. 7 except for the upper quasienergy states.

It becomes more difficult for the electron to jump from one potential well to the other as $R$ increases. Finally, within each optical cycle, there are small fluctuations. These may be attributed to the charge resonance enhanced multiphoton excitation.

Now we come to the center of the question regarding why there exists two ionization peaks for both lower and higher quasienergy states seen in Fig. 3. Using the dc-field picture, Shakeshaft et al., [8] argued that the ionization enhancement of the " $2 \mathrm{p}$ " state is due to its mixing with energetically nearby highly excited states that are localized in the lower electronic-field potential well, followed by the over-thebarrier ionization from the lower well. In the following we explore the mixing of the bare $1 \sigma_{g}$ and $1 \sigma_{u}$ states with excited electronic states in the presence of the low-frequency ac field. This provides new insights regarding the actual mechanism responsible for the ionization enhancement. Recall that we have found earlier that the majority of the electron population remains in the $1 \sigma_{g}$ and $1 \sigma_{u}$ states. However, these two levels alone cannot contribute to the ionization. In reality, they have some substantial mixing with excited electronic states through various multiphoton resonances. Since these excited electronic states have much larger ionization widths than those of $1 \sigma_{g}$ and $1 \sigma_{u}$ states, their contributions can lead to the overall ionization enhancement. To explore this further, we plot in Fig. 9 the electron population of various excited electronic states estimated by the projection of the lower quasienergy state onto various field-free excited electronic states. Figure 9 shows that at $R$ around $9 \mathrm{a}_{0}$, there are substantial populations of various excited electronic states, with the major contribution arising from the $3 \sigma_{g}$ state at $R \simeq 9 \mathrm{a}_{0}$. This accounts for the major ionization enhancement at $R \simeq 9 \mathrm{a}_{0}$ of the lower quasienergy state seen in Fig. 3. Similarly, the other smaller ionization enhancement of the lower quasienergy state is due to the contributions of the excited states around $R \simeq 6 \mathrm{a}_{0}$ with the dominant contributions arising from the $2 \sigma_{g}$ and $4 \sigma_{g}$ states, etc. Figure 10 shows the corresponding information for the "upper", quasienergy state. Similar analysis provides the clue to the 

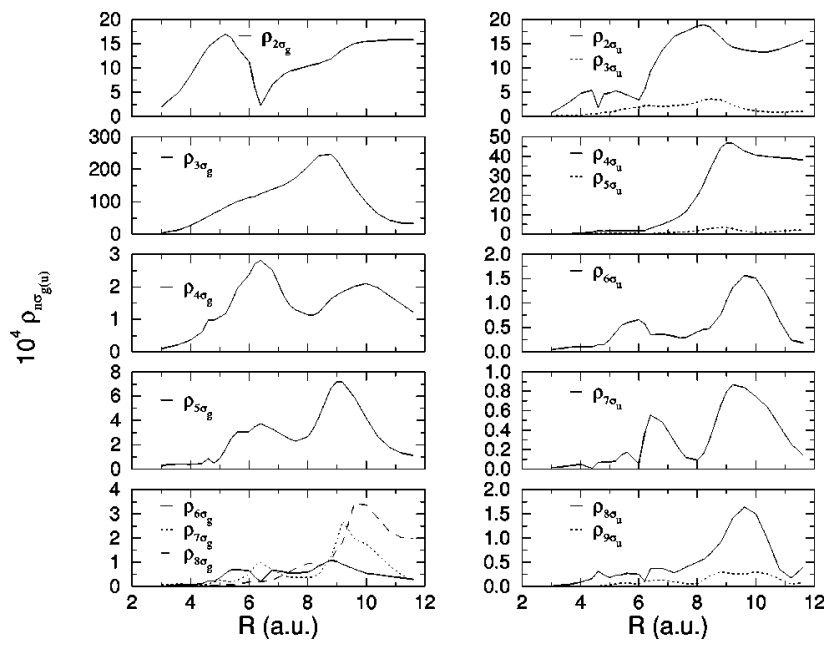

FIG. 9. The electron population of various excited electronic states calculated from the lower quasienergy states.

ionization enhancement at $R \simeq 8 \mathrm{a}_{0}$ and $5 \mathrm{a}_{0}$ of the upper quasienergy state seen in Fig. 3.

Finally it is instructive to make some comparison of the present time-independent Floquet results with other results such as those obtained by the numerical solution of the timedependent Schrödinger equation [6]. In general one expects that the time-dependent calculations can reproduce the behavior of Floquet quasienergy results if both calculations are fully converged and if the following two conditions are satisfied: (a) the laser field is turned on adiabatically, and (b) there are no avoided crossings of quasienergy levels. Indeed excellent agreements of the time-dependent and Floquet results were obtained in a number of previous studies of multiphoton ionization and/or high harmonic generation rates of atomic systems [21]. In the time-dependent calculation of $\mathrm{H}_{2}^{+}$by Zuo and Bandrauk [6], the laser field is turned on linearly in the first five optical cycles and then kept constant. The norm of the wave function decays exponentially due to the removal of the electron at the boundary of the numerical grid. The overall feature of the time-dependent results [6] is
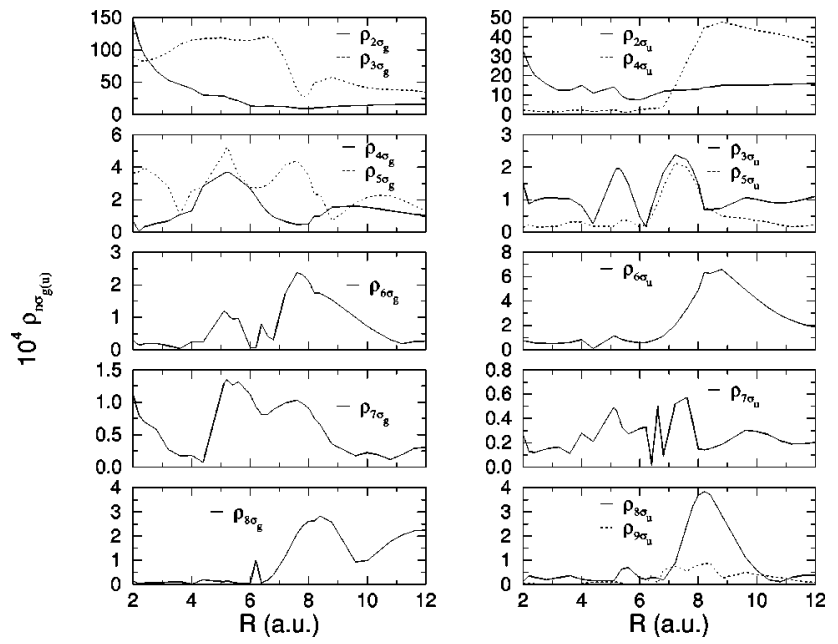

FIG. 10. The electron population of various excited electronic states calculated from the upper quasienergy states.

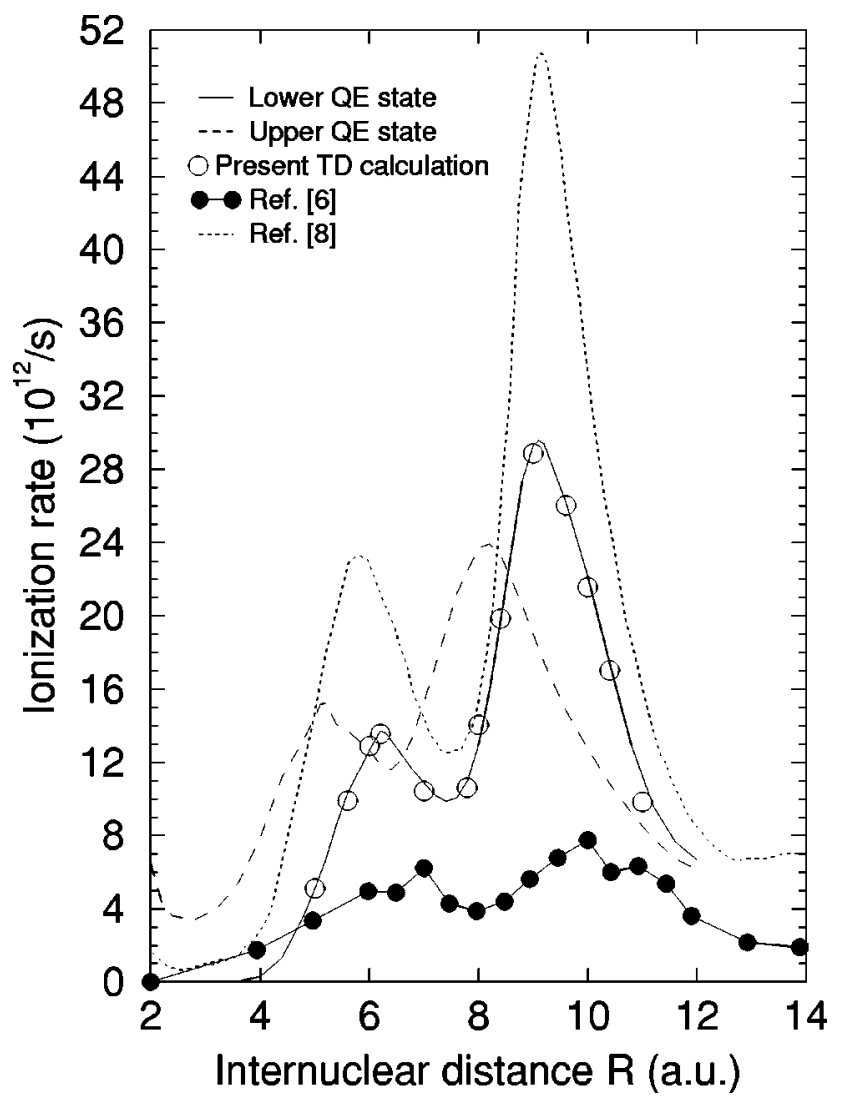

FIG. 11. Comparison of the ionization rate of $\mathrm{H}_{2}^{+}$vs $R$ obtained by the time-dependent numerical calculations of Ref. [6] and by the present time-independent Floquet quasienergy (QE) calculations. Also shown here for comparison are our new time-dependent (TD) results (open circles) and the cycle-averaged dc quasienergy widths of the $2 p$ state (dotted line) of Ref. [8]. The laser wavelength and intensity used are $1064 \mathrm{~nm}$ and $10^{14} \mathrm{~W} / \mathrm{cm}^{2}$, respectively.

qualitatively similar to the pattern of the lower or upper quasienergy level (also reproduced in Fig. 11 for comparison), showing the ionization enhancement can occur at two larger internuclear distances. The peak positions of the timedependent calculations [6] are closer to those of the lower quasienergy level. However, the peak ionization rates of Ref. [6] are smaller than those of the lower quasienergy level. The situation is somewhat similar to that shown in Table I, where the dc widths obtained by Ref. [6] are also smaller by a factor of about 3 than our results and those of Ref. [8].

Also shown in Fig. 11 for comparison are the the cycleaveraged dc quasienergy widths of the $2 p$ state (i.e., the average of the dc ionization rates over all instantaneous value of the ac-field strength during one optical cycle) obtained by Mulyukov et al., [8]. These (frequencyindependent) dc quasienergy widths are significantly higher than those of the time-dependent results of Zuo and Bandrauk [6], although the peak heights of the dc rates are likely to be an overestimation. Mulyukov et al., [8] also considered the $\omega^{2}$ correction term to their dc quasienergy widths, but the perturbative correction breaks down near the peak positions.

A couple of intriguing questions arise regarding the comparison of the time-dependent calculations with the present Floquet results. Do both lower and upper quasienergy levels 
contribute to the total rate in the time-dependent calculations? What happens if the field is turned on adiabatically? Also can the near degeneracy of the unperturbed $1 \sigma_{g}$ and $1 \sigma_{u}$ states at intermediate and large distances cause some mixing of the two quasienergy levels in the time-dependent calculations? To answer some of these questions, we have performed a time-dependent calculation independently by means of the time-dependent generalized pseudospectral (TDGPS) method recently developed in our group for nonperturbative treatment of multiphoton ionization and high harmonic generation (HHG) of atoms [20] and molecules [22] in strong fields. The TDGPS procedure allows nonuniform and optimal spatial grid discretization of the Hamiltonian, which is essential for the Coulomb potential, and efficient time propagation of the wave function. Highprecision time-dependent wave functions can be obtained by this procedure as demonstrated, for example, by the excellent agreement of the HHG power spectra in length and acceleration forms over 20 orders of magnitude [20,22]. In the present time-dependent calculation, the field is turned on linearly for the first ten optical cycles and then the field amplitude is kept constant. The initial state at $t=0$ is assumed to be in the unperturbed $1 \sigma_{g}$ electronic state of $\mathrm{H}_{2}{ }^{+}$. Our timedependent results for the intermediate and large distances are shown by the open circles in Fig. 11. interestingly, the ionization rates from our time-dependent calculations follow closely and nearly coincide with those of the lower quasienergy level. This indicates that our field turn-on procedure appears "adiabatic" enough and the lower quasienergy level unambiguously provides all the needed information for accounting the enhanced ionization at intermediate and large $R$. The agreement of our time-dependent and Floquet results also confirms the numerical convergency of both calculations. Note that in the range of internuclear distances $(R$
$=5$ to $12 \mathrm{a}_{0}$ ) in our time-dependent calculations, there is no avoided crossing of the upper and lower quasienergy levels (cf. Fig. 2). The upper quasienergy level is likely to also contribute to the ionization rate in the avoided crossing regions in nonadiabatic time-dependent calculations. In summary, the analysis of the quasienergy level behavior presented in this paper provides new insights regarding the detailed ionization enhancement mechanisms, which cannot be easily obtained by the time-dependent procedure.

In conclusion, we have presented a new CSGPS procedure for accurate and efficient determination of the complex quasienergy states of two-center molecular systems. We have applied the procedure to the study of the mechanism responsible for the ionization enhancement of $\mathrm{H}_{2}{ }^{+}$in intense lowfrequency $(1064 \mathrm{~nm})$ laser fields. From the present Floquet study, we lead to the conclusion that the ionization enhancement is mainly due to the charge resonance enhanced multiphoton resonances of the $1 \sigma_{g}$ and $1 \sigma_{u}$ states with excited electronic states. The "critical" distances where the ionization enhancement occur depend on the molecular electronic structure and laser frequency and intensity used. Finally, we note that the outlined CSGPS procedure in this paper is quite general and can be applied to other two-center problems as well. We are currently extending the CSGPS method along with the Floquet formulation of time-dependent densityfunctional theory [21] for nonperturbative treatment of multiphoton processes of many-electron systems in intense laser fields.

\section{ACKNOWLEDGMENTS}

This work is partially supported by NSF under Grant No. 9801889. We acknowledge Kansas Center for Advanced Scientific Computing for the support of Origin2400 supercomputer time.
[1] H. Stapelfeldt et al., Phys. Rev. A 58, 426 (1998).

[2] E. Constant, H. Stapelfeldt, and P.B. Corkum, Phys. Rev. Lett. 76, 4140 (1996).

[3] G.N. Gibson, M. Li, C. Guo, and J. Neira, Phys. Rev. Lett. 79, 2022 (1997).

[4] K. Codling and L.J. Frasinski, J. Phys. B 26, 783 (1993).

[5] J.H. Posthumus et al., J. Phys. B 29, L525 (1996).

[6] T. Zuo and A.D. Bandrauk, Phys. Rev. A 52, R2511 (1995).

[7] M. Plummer and J.F. McCann, J. Phys. B 29, 4625 (1996).

[8] Z. Mulyukov, M. Pont, and R. Shakeshaft, Phys. Rev. A 54, 4299 (1996).

[9] T. Seideman, M.Yu. Ivanov, and P.B. Corkum, Phys. Rev. Lett. 75, 2819 (1995).

[10] G. Yao and S.I. Chu, Chem. Phys. Lett. 204, 381 (1993).

[11] J. Wang, S.I. Chu, and C. Laughlin, Phys. Rev. A 50, 3208 (1994).

[12] See, for example, D. Telnov and S.I. Chu, Phys. Rev. A 59, 2864 (1999), and references therein.

[13] D.A. Telnov, J. Wang, and S.I. Chu, Phys. Rev. A 52, 3988 (1995).
[14] M. R. Spiegel, Mathematical Handbook (McGraw-Hill, New York, 1997).

[15] See, for example, A.D. Becke, J. Chem. Phys. 76, 6037 (1982).

[16] M.M. Madsen and J.M. Peek, At. Data 2, 176 (1971).

[17] E. Balslev and J.M. Combes, Commun. Math. Phys. 22, 280 (1971); A. Aguilar and J.M. Combes, ibid. 22, 265 (1971).

[18] For the reviews the on non-Hermitian Floquet methods, see S.I. Chu, Adv. At. Mol. Phys. 21, 197 (1985); Adv. Chem. Phys. 73, 739 (1991).

[19] R.S. Mulliken, J. Chem. Phys. 7, 20 (1939).

[20] See, for example, X.M. Tong and S.I. Chu, Chem. Phys. 217, 119 (1997).

[21] See, for example, D.A. Telnov and S.I. Chu, Chem. Phys. Lett. 264, 466 (1997); Phys. Rev. A 58, 4749 (1998).

[22] For a discussion of the extension of the TDGPS method to the two-center molecular systems, see X. Chu and S. I. Chu, Multiphoton Processes, edited by L. F. DiMauro, R. R. Freeman, and K. C. Kulander (American Institute of Physics, New York, 2000), p. 415. 